\title{
Investigation of Magnetic Guidance of Cochlear Implants
}

\author{
James R. Clark, Lisandro Leon, Frank M. Warren, and Jake J. Abbott
}

\begin{abstract}
Cochlear implants have become a standard treatment for many with severe to profound sensorineural hearing loss. However, delicate cochlear structures can be damaged during surgical insertion, which can lead to loss of residual hearing and decreased implant effectiveness. We propose a magnetic guidance concept in which a magnetically tipped cochlear implant is guided as it is inserted into the cochlea. In a scaled in vitro experimental study, we record insertion forces for nonguided and magnetically guided insertion experiments and compare the results. Results indicate that magnetic guidance reduced insertion forces by approximately $50 \%$.
\end{abstract}

\section{INTRODUCTION}

A cochlear implant (CI) is an array of electrodes embedded in silicone that is surgically inserted into the scala tympani (ST) chamber of the cochlea to electrically stimulate the nerves responsible for hearing (Fig. 1). Cochlear implants have become a standard treatment for many with severe to profound sensorineural hearing loss. However, the majority of CI users still have difficulty understanding speech in an environment with competing noise and difficulty distinguishing a full range of sounds. Future CIs will need to address three widely accepted goals [1]: (1) deeper insertion into the ST to access lower frequency cochlear neurons, (2) greater operating efficiency, defined as a reduction in the stimulus charge required to produce a comfortable loudness level, and (3) reduced intracochlear damage during surgical insertion.

As the electrode array is inserted into the ST, delicate intracochlear structures are often damaged, which can result in loss of residual hearing and decreased implant effectiveness, especially when the implant deviates into another cochlear chamber [1]. Two major factors that contribute to the extent of damage during insertion are electrode-array design and surgical technique [2]. Numerous electrode-array designs have been developed with varied success [1]-[4], and to date, no single design has been able to achieve all three objectives of deeper insertion, proximity to the modiolus, and consistent atraumatic insertion [1]. Studies comparing the insertion techniques for precurved CIs with stylets have concluded that the standard insertion technique generally resulted in the highest insertion forces while the advance off-stylet technique showed a notable decrease in forces for nearly the entire insertion procedure [5]-[7]. However, advancing the CI off the stylet too early could cause the implant tip

This work was supported by the National Science Foundation under grants IIS-0952718 and DGE-0654414.

J. R. Clark, L. Leon, and J. J. Abbott are with the Department of Mechanical Engineering, University of Utah, Salt Lake City, UT 84112 \{james.r.clark, l.leon, jake.abbott\}@utah.edu

F. M. Warren is with the Department of Otolaryngology, Division of Otology and Skull Base Surgery, Oregon Health \& Science University, Portland, OR 97239 warrenf@ohsu. edu

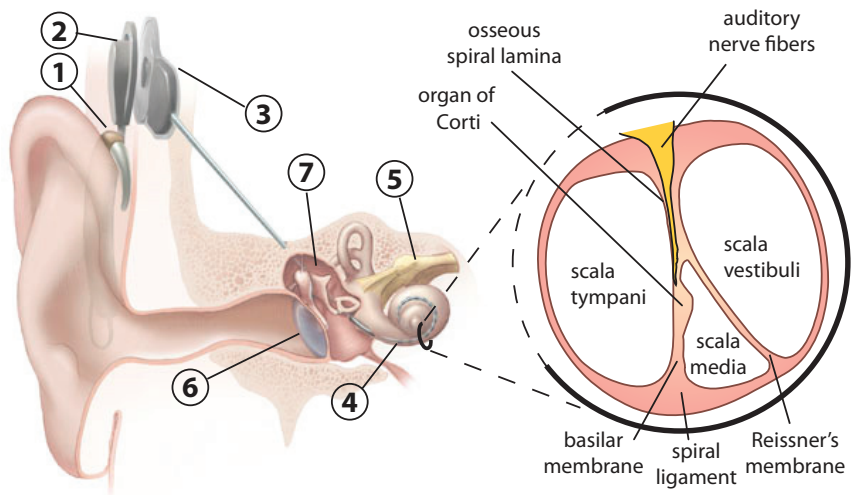

Fig. 1. Cochlear implant system with blow-up of cochlea cross section showing the location of several cochlear structures $(\mathrm{NIH}$ public domain image with added labels). Labeled items are the (1) microphone and speech processor, (2) transmitter, (3) receiver, (4) electrode array inserted into the cochlea (referred to as the "cochlear implant" herein), (5) auditory nerve, (6) ear drum, and (7) ossicles.

to fold-over, and late stylet removal could result in the implant contacting the ST outer wall, potentially causing damage. The variability in human cochlear dimensions poses a challenge when using stylet withdrawal techniques, since the point during insertion when stylet removal should begin is patient dependent [1].

Due to the limitations of existing CIs, several groups have developed electrode-array prototypes to achieve a perimodiolar position within the cochlea [8], with some actively bent or steered during insertion to minimize insertion trauma [9][12]. Some designs [9], [11], [12] use mechanical means built into the $\mathrm{CI}$ to achieve bending and can increase the stiffness of the $\mathrm{CI}$ as it is being inserted. If the $\mathrm{CI}$ is not formed to fit the ST well or is misdirected down the channel, the increased CI stiffness could result in increased intracochlear trauma. Others are not reversible [8], [11] or require sufficient perilymph fluid to actuate [8], [10], which may be problematic if, during surgery, reinsertion is necessary [2] or sufficient fluid is lost. Finally, the wide variability in surgical force application has motivated the use of robotic assistance [13]-[15] and optimized path planning [12] to produce more repeatable insertions.

In this paper, we propose a magnetic guidance concept in which a magnetically tipped CI is guided as it is inserted into the cochlea. With a rotating manipulator magnet located near the patient's head, we apply magnetic torque to the implant tip, causing it to bend away from the ST walls during insertion. We conduct proof-of-concept experiments of two proposed magnetic guidance methods, using an automated experimental apparatus to insert a scaled magnetically tipped implant prototype into a custom 3:1 scale ST phantom [16]. 


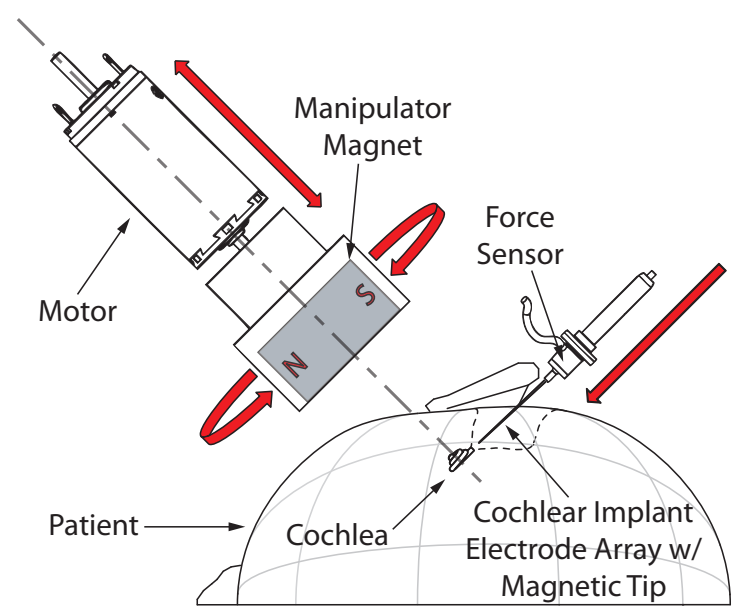

Fig. 2. Concept for magnetically guided cochlear implant surgery. Red wide arrows indicate the three controlled degrees of freedom.

We record insertion forces during nonguided and magnetically guided insertion experiments and compare the results. The data show that magnetic guidance can reduce insertion forces by approximately 50\%. Our method is consistent with the current practice of using insertion force measurements (which encode implant-ST contact forces) as a metric for evaluation of insertion trauma [5]-[7], [12]-[14]. Our approach is an adaptable means of guidance that could be robotically implemented as either a supervised automated process or a robotic system under surgeon manipulation. The basic idea of magnetic guidance for CIs has been proposed previously [17], but no technical results were presented to demonstrate how it could be accomplished in practice.

\section{Magnetic Guidance Concept}

A clinical concept for magnetically guided CI insertions is shown in Fig. 2. To achieve CI guidance, a small permanent magnet is located at the tip of the implant. A large manipulator magnet located near the patient's head is used to apply a magnetic field to the CI tip. The manipulator magnet's orientation is controlled by a motor, with the magnet's magnetization direction perpendicular to the axis of rotation. The rotation axis is approximately aligned with the central spiral axis of the cochlea. As the CI is inserted, the manipulator magnet is rotated to actively bend the implant, directing it away from cochlear walls and reducing the contact forces between the CI and the walls of the ST. The motor is allowed to translate along its rotation axis, varying the distance between the manipulator magnet and the patient, effectively changing the strength of the applied magnetic field acting on the CI tip. The CI insertion is automated and synchronized with the movement of the manipulator magnet in our developed control software.

The field $\mathbf{B}(\mathrm{T})$ generated by the manipulator magnet, modeled as a magnetic dipole $\mathbf{M}\left(\mathrm{A} \cdot \mathrm{m}^{2}\right)$, can be approximated by the point-dipole model

$$
\mathbf{B}(\mathbf{p})=\frac{\mu_{0}}{4 \pi|\mathbf{p}|^{3}}\left(\frac{3(\mathbf{M} \cdot \mathbf{p}) \mathbf{p}}{|\mathbf{p}|^{2}}-\mathbf{M}\right)
$$

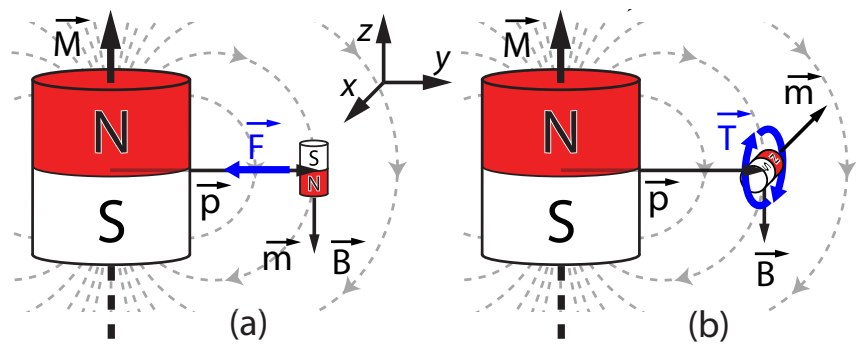

Fig. 3. Magnetic force and torque in clinical arrangement of Fig. 2. The large magnet is the manipulator magnet, with the coordinate frame origin at its dipole center (shown offset for clarity). The implant tip magnet is placed along the manipulator's rotation axis making $\mathbf{M}$ and $\mathbf{p}$ orthogonal. Two directions of the implant tip magnet with resulting forces and torques are shown. (a) Negative $\mathrm{z}$-direction $\left(\theta=0^{\circ}\right)$ : force in negative y-direction. (b) Negative $\mathrm{x}$-direction $\left(\theta=90^{\circ}\right)$ : negative torque about $\mathrm{y}$-axis.

where $\mu_{0}$ is the permeability of free space $\left(\mu_{0}=4 \pi \times\right.$ $10^{-7} \mathrm{~T} \cdot \mathrm{m} \cdot \mathrm{A}^{-1}$ ) and $\mathbf{p}$ is the location of a point in space with respect to the magnet's center $(\mathrm{m})$. A magnet's dipole strength is the product of its volume $\left(\mathrm{m}^{3}\right)$ and its average magnetization $(\mathrm{A} / \mathrm{m})$. Since the field is nonuniform, magnetic forces and torques will act upon any magnet placed within this nonuniform field. If we represent the permanent magnet embedded in the CI's tip by the dipole $\mathbf{m}$, then the force and torque acting on it due to the manipulator magnet are

$$
\begin{gathered}
\mathbf{F}=\left[\begin{array}{ccc}
\frac{\partial}{\partial x} \mathbf{B} & \frac{\partial}{\partial y} \mathbf{B} & \frac{\partial}{\partial z} \mathbf{B}
\end{array}\right]^{\mathrm{T}} \mathbf{m} \\
\mathbf{T}=\mathbf{m} \times \mathbf{B}
\end{gathered}
$$

where $\mathbf{B}$ is the field at the location of $\mathbf{m}, \mathbf{F}$ is force $(\mathrm{N})$, and $\mathbf{T}$ is the torque $(\mathrm{N} \cdot \mathrm{m})$, expressed in the same frame in which the spatial derivatives are taken [18]. From (2) we see that the force in a given direction is the inner product of the derivative of the field in that direction and the magnetization of the magnet placed in the field. This means that a force will only act on the magnet in a given direction if both a field gradient and some component of $\mathbf{m}$ exist in that direction. The applied torque is the cross product of the magnet's magnetization with the applied field, meaning that a torque will only act on the magnet if its dipole is not parallel to the applied field.

Figure 3 demonstrates how our clinical configuration allows (1)-(3) to be simplified. Note the CI magnet is positioned approximately on the manipulator magnet's rotation axis, and can be rotated about the $\mathrm{y}$-axis in the $\mathrm{xz}$-plane to any rotation angle $\theta$ between $\mathbf{m}$ and the local $\mathbf{B}$. The vector $\mathbf{p}$ points from the manipulator magnet to the $\mathrm{CI}$ magnet. As the manipulator magnet is moved closer to or farther from the patient along the manipulator's rotation axis, only the field strength changes. Thus, moving the manipulator toward or away from the patient will change the strength of the field applied at the CI tip, but the field direction will not change unless the manipulator magnet is rotated. Since CI tip rotations are largely confined to the $\mathrm{xz}$-plane within the ST channel, the torque of (3) simplifies to

$$
|\mathbf{T}|=|\mathbf{m}||\mathbf{B}| \sin (\theta) .
$$

Maximum torque occurs when the dipoles are perpendicular to one another; when the dipoles are aligned, the torque is 
zero. The general equation for the field strength in (1) and its gradient also simplify along the axis of the motor:

$$
\begin{gathered}
|\mathbf{B}|=\frac{\mu_{0}|\mathbf{M}|}{4 \pi|\mathbf{p}|^{3}} \\
\frac{d|\mathbf{B}|}{d|\mathbf{p}|}=-\frac{3 \mu_{0}|\mathbf{M}|}{4 \pi|\mathbf{p}|^{4}}
\end{gathered}
$$

Furthermore, after some manipulation, the magnetic force of (2) can be expressed in a form that more clearly shows its behavior when $\mathbf{m}$ is located along the rotation axis:

$$
\mathbf{F}=\frac{d|\mathbf{B}|}{d|\mathbf{p}|}\left[\begin{array}{ccc}
0 & 0 & 0 \\
0 & 0 & -1 \\
0 & -1 & 0
\end{array}\right] \mathbf{m}
$$

We see from (7) that no gradient exists in the $x$-direction (first row), whereas gradients do exist in the $\mathrm{y}-$ and $\mathrm{z}-$ directions (second and third rows, respectively). Since there is no $\mathrm{x}$-direction gradient, forces will not act on the magnet in the $\mathrm{x}$-direction for any $\mathbf{m}$. The second row indicates that a force will only act in the y-direction if some component of $\mathbf{m}$ points in the $\mathbf{z}$-direction. This force reaches a maximum magnitude when $\mathbf{m}$ is completely aligned with the $\mathbf{z}-$ direction, as shown in Fig. 3(a). The third row indicates that a force will only act in the $\mathrm{z}$-direction if some component of $\mathbf{m}$ points in the $\mathbf{y}$-direction; however, since the CI tip is confined to rotate largely in the xz-plane, the components of $\mathbf{m}$ point largely in the $\mathbf{x}-$ and $\mathbf{z}$-direction. Thus, we assume that the $\mathrm{z}$-direction force is negligible. In practice, the CI tip rotation will not be strictly confined to the $\mathrm{xz}$-plane, but the minor deviations we expect should only result in minor forces and torques along directions not shown in Fig. 3.

We have devised two control algorithms to synchronize the motions of the manipulator magnet and the CI insertion (Fig. 4). The first is the maximum-field method, in which the manipulator magnet is placed as close to the patient as is physically possible for the duration of the insertion, and is only allowed to rotate. In this method, the magneticfield magnitude is always at its maximum, and the CI tip is nearly aligned with the manipulator magnet. This algorithm is desirable in that it does not require the use of a linear stage to vary the manipulator magnet's position. Unfortunately, the CI tip experiences attractive forces toward the manipulator magnet, which contributes to the force on the basilar membrane that we would ultimately like to reduce. The second algorithm is the maximum-torque method, in which the applied field is always maintained approximately perpendicular to the CI tip, resulting in a pure magnetic torque with negligible attractive forces. This method requires variable distance between the manipulator magnet and $\mathrm{CI}$ in order to control the required field magnitude.

\section{ExPerimental Methods}

To validate the basic magnetic-guidance method, we constructed an automated experimental apparatus (Fig. 5) consisting of a CI prototype, a force sensor, custom mounting
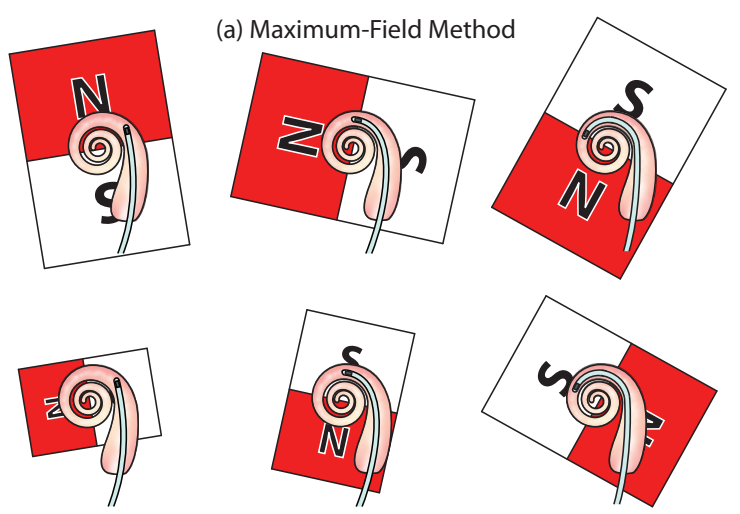

(b) Maximum-Torque Method

Fig. 4. Two guidance methods explored in experiments. (a) Maximum-field method: the dipoles are nearly aligned. (b) Maximum-torque method: the dipoles are nearly perpendicular. The increasing manipulator magnet size in the maximum-torque diagram indicates that the manipulator is advancing toward the cochlea.

fixtures, an ST phantom, linear stages, a manipulator magnet, a servo system (not labeled), and a standard PC (not shown).

We constructed a prototype CI with a small $(1 \mathrm{~mm}$ diameter $\mathrm{x} 2 \mathrm{~mm}$ long) permanent magnet (of approximate dipole strength $2 \mathrm{~mA} \cdot \mathrm{m}^{2}$ ) embedded in its tip; the prototype is a 3:1 scale model of a free-fitting straight CI. Our goal was not to duplicate a scaled version of a commercial CI, but to develop one that could be used to validate our magnetic guidance concept. We also wanted the prototype to qualitatively mimic some important properties of a real CI (such as the MEDEL practice electrode in Fig. 6), including graded stiffness, bending properties, and the use of silicone rubber. The process to fabricate the prototypes is shown in Fig. 7.

We inserted the implant into a custom 3:1 scale scalatympani phantom filled with soap solution; the phantom's model, described in detail in [16], is based on published anatomical data, and can be manufactured to a desired scale.

Thorlabs linear motorized stages were used to insert the $\mathrm{CI}$, as well as to control the position of the manipulator magnet with respect to the phantom. The manipulator was attached to the MTS50/M-Z8 linear stage mounted horizontally to the table. Two MTS50/M linear stages used to insert the implant were mounted vertically in series to increase the total translational range, allowing for deeper implant insertions into the ST phantom.

An ATI Nano17 six-axis force/torque sensor was used to collect force data during insertion experiments. Along the sensor $\mathrm{z}$-axis, the minimum resolution is $3.125 \mathrm{mN}$ and the maximum measurement uncertainty is $170 \mathrm{mN}$, which is the maximum amount of error in any single measurement. However, under certain loading conditions, the sensor performs significantly better. In the accompanying calibration report, measurement uncertainty of $1.7-3.4 \mathrm{mN}$ was reported for sensor loading along its z-axis exclusively, which is the loading condition of our experimental setup.

Custom mounting fixtures, printed in ABSplus ${ }^{\mathrm{TM}}$ thermoplastic using a Dimension 3D printer, were used to attach the force sensor to the Thorlabs mounting posts and the implant prototype to the front (tool side) of the sensor. 


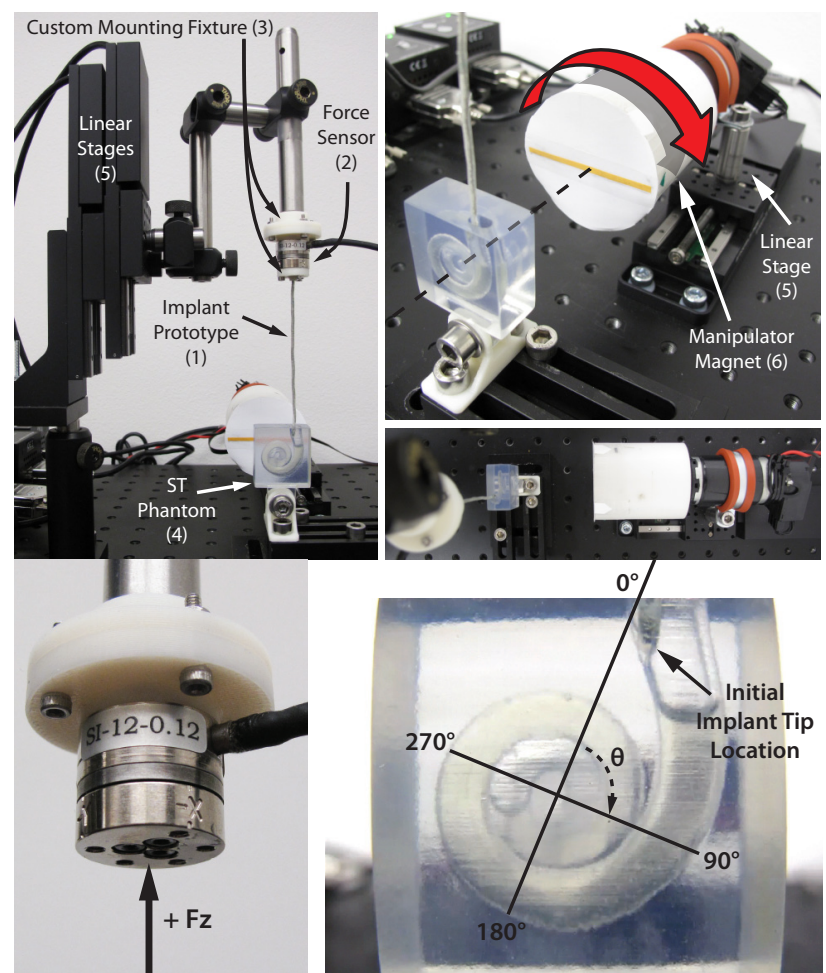

Fig. 5. Experimental setup. (top) The prototype (1) was attached to a force/torque sensor (2) with custom mounting fixtures (3) and inserted into a phantom (4) using linear stages (5). The manipulator (6) is attached to a brushed DC motor shaft and mounted to a linear stage, which translates the manipulator towards the phantom. The dashed line shows that the manipulator rotation axis and cochlear central spiral axis are aligned. The curved arrow above the manipulator shows the manipulator rotation direction. The stripe on the front of the manipulator marks the dipole direction of the enclosed axially magnetized magnet. (bottom-left) ATI Nano17 force/torque sensor with definition of positive force. (bottom-right) Insertion angle based on the phantom model [16].

The manipulator consists of a Delrin housing attached to a motor shaft with a $25.4-\mathrm{mm}$-diameter $\times 25.4-\mathrm{mm}-$ long $\mathrm{NdFeB}$ permanent magnet placed in the housing. The cylindrical magnet is axially magnetized with a measured dipole strength of $10.2 \mathrm{~A} \cdot \mathrm{m}^{2}$.

The rotation of the manipulator magnet was controlled using commercial servo components including a digital servo drive (Advanced Motion Controls DigiFlex Performance DPR Series), DC power supply (GW Instek GPS-3303), and a brushed DC motor and encoder unit (Maxon A-max 32 and HEDS 5540). The motion of the CI was captured through the transparent phantom using a commercial camera (Canon PowerShot G10). System control and data logging were performed with a PC running Windows.

The experiments were conducted on a Thorlabs metric optical table with the following arrangement. The implant prototype was mounted to the vertically stacked stages oriented so that the prototype pointed downward. The 3:1 ST phantom was attached vertically to the table so that the entrance of the chamber was facing upward, with the ST chamber spiraling toward the manipulator magnet. Adjustments were made so that the manipulator rotation axis was approximately aligned with the central spiral axis of the cochlea.

The sensor itself experiences forces and torques from the

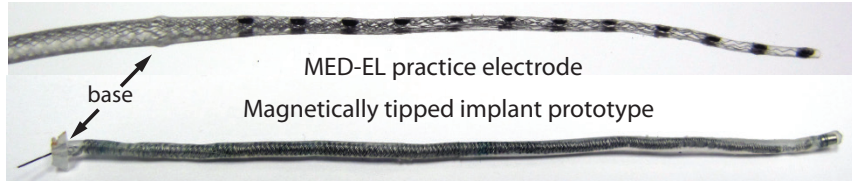

Fig. 6. (top) The MED-EL practice electrode shown is a straight, freefitting electrode array with a length of $32 \mathrm{~mm}$, and it tapers from a base diameter of $1.3 \mathrm{~mm}$ to a tip diameter of $0.5 \mathrm{~mm}$. (bottom) Our 3:1 prototype has a length of $83 \mathrm{~mm}$ and a constant diameter of $1.6 \mathrm{~mm}$.

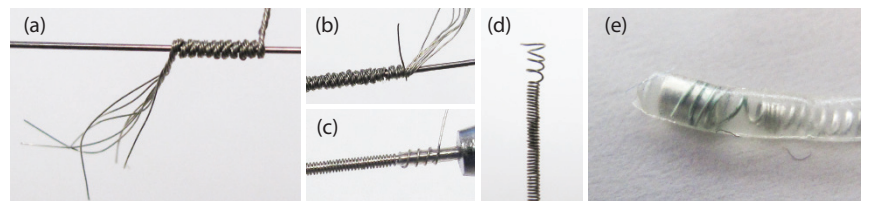

Fig. 7. Key steps for production of the wire core used in the implant prototype. (a) Wires twisted together and wrapped around a cylindrical shaft. (b) Snip off one wire at a certain length and wrap the remaining wires. Repeat this process until only one wire is left. This creates a tapered stiffness, which was observed in the MED-EL device. (c) Wrap remaining wire around tubing to create a coil that the magnet can be slid into. (d) The result of the wire wrapping prior to placing the magnet. (e) Magnet is placed at the end of the coil and encased in silicone using an acrylic mold.

manipulator magnet since the sensor is made of ferromagnetic material. Thus, to calibrate for these effects, insertion measurements were taken while running experiments without the CI prototype attached to the sensor. Five calibration runs were averaged and subtracted from the insertion measurements recorded with the prototype attached.

We conducted nonguided and magnetically guided experiments on the same CI, with all other factors held constant. During the experiments, the closest distance between the manipulator magnet and the CI tip corresponded to a dipole to dipole distance of $29 \mathrm{~mm}$. For the magnetically guided insertions, we used both maximum-field and maximumtorque methods described earlier. Linear stage translations and manipulator magnet rotations were experimentally predetermined so that the CI's tip always stayed parallel to the channel throughout the experiment. At each discrete position in $1 \mathrm{~mm}$ intervals along this trajectory, $10 \mathrm{~ms}$ of force data, sampled at $1 \mathrm{kHz}$, was collected and averaged.

\section{EXPERIMENTAL RESULTS}

The measured insertion forces (along the sensor z-axis) are shown in Fig. 8(a). The variance between runs was calculated and presented as solid vertical lines indicating the 95\% $(2 \sigma)$ confidence interval for each data point. Insertion depths are measured from the initial implant position shown in Fig. 5. The vertical dashed lines show the approximate insertion depths where the implant first made contact with the phantom outer wall. Their corresponding images are shown in Fig. 8(b). For the nonguided insertions, outerwall contact first occurred at $17.5 \mathrm{~mm}$. The use of magnetic guidance clearly allows the CI to be inserted deeper into the ST phantom before it makes initial contact. For an insertion depth between $0 \mathrm{~mm}$ and $25 \mathrm{~mm}$, no method shows a clear advantage. However, the magnitudes of the forces in this region are so small that they are of limited interest. After $25 \mathrm{~mm}$, the nonguided approach is inferior to both 


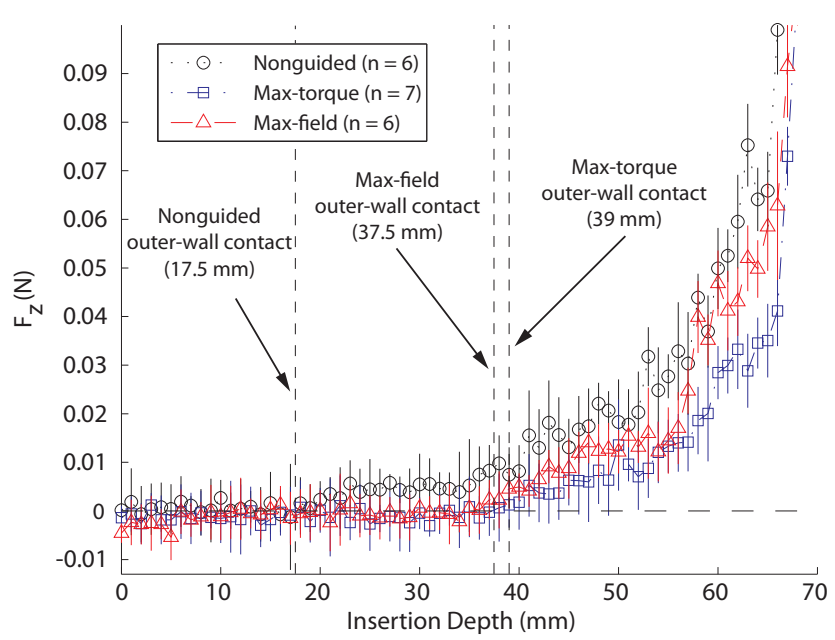

(a)

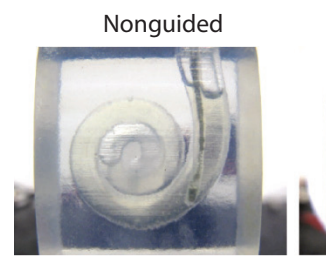

$17.5 \mathrm{~mm}$

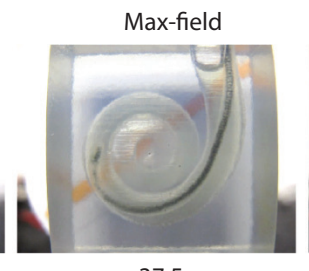

$37.5 \mathrm{~mm}$

(b)

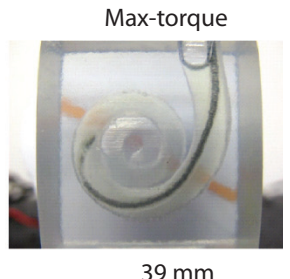

$39 \mathrm{~mm}$

Fig. 8. (a) Experimental insertion-force data $\left(F_{z}\right)$ for all three insertion methods. Each data point represents the average of $n$ runs where the collected measurement for each experiment is an average of 10 samples at the corresponding insertion depth. The solid vertical bar with each data point shows the 95\% (2 $\sigma)$ confidence interval. Vertical dashed lines mark the approximate locations where the implant first made contact with the ST outer wall. (b) Corresponding images showing implant positions at first instance of outer-wall contact.

magnetically guided strategies. As long as the prototype avoids contact with the ST, insertions forces are negligible, and although the guided implant eventually slides against the ST outer wall, the CI touches the wall softer when using guidance, and the tip is bent away from the wall, decreasing both frictional and tip contact forces. In general, nonguided insertions resulted in the highest force and the maximum-torque method generally resulted in the lowest force. These results show that insertion forces can be reduced by approximately $50 \%$ using magnetic guidance. This is consistent with our hypothesized outcome described in Section II: the maximum-torque method minimizes attractive forces between the CI and the manipulator magnet, which in turn minimizes lateral scraping on the ST wall. These initial results are promising, indicating that significant insertionforce reduction is possible using magnetic guidance.

\section{Discussion}

Without guidance, the inherent stiffness in a free-fitting CI produces a mechanical restoring torque that presses the $\mathrm{CI}$ against the outer ST wall, resulting in increased friction and insertion force. With magnetic guidance, the torque acting on the CI tip counteracts this mechanical restoring torque. If the magnetic torque is sufficient, the CI will be directed completely away from the wall. If not, the magnetic torque

will still provide some decrease in contact forces.

Our straight, free-fitting prototype limits the benefit of magnetic guidance since eventually the applied magnetic torque cannot overcome the natural tendency of the prototype to maintain its relaxed, straight shape. If the CI were fabricated so that its relaxed shape approximated the ST curvature (as is done with some current clinical CIs) then we could use our magnetic guidance concept to uncoil these precurled CIs during insertion and conceivably achieve nearzero insertion force throughout the insertion. Furthermore, close proximity between the $\mathrm{CI}$ and the modiolus reduces the power consumption required by the electrodes and the crosstalk responsible for poor frequency resolution due to one electrode stimulating multiple locations on the nerve. This strategy could be easily adapted to improve stylet removal methods (such as advance off-stylet) by straightening the CI during the critical moments when withdrawing the stylet can result in the CI curving into the ST inner wall.

Real CIs have a tapered shape; that is, they are thicker at the base and thinner at the tip. Our prototype has a constant diameter, which precludes insertions into our ST phantom greater than $560^{\circ}$. Tapering the CI to have a tip with a smaller diameter (and a smaller embedded magnet) would allow deeper insertion into the ST, and the tapered shape would help provide the necessary basal stiffness to avoid buckling. We plan to create a CI prototype that addresses these limitations. Our future prototype will have a relaxed shape that hugs the modiolus (based on our ST model [16]). It will also have a tapered CI profile, along with the desired bending properties, such that a magnetic torque applied at the tip results in the most desirable uncoiled shape for insertion.

In the experiments herein, we used force sensing only for analysis after the insertion had been performed. Others have used force feedback to regulate insertion speed during their experiments using a steerable prototype electrode array [12]. We plan to utilize force measurements in real-time and develop control algorithms that minimize insertion forces adaptively throughout the insertion, essentially allowing the CI to "feel around in the dark," as opposed to relying on medical imaging for guidance. This capability would be advantageous since preoperative imaging to determine the dimensions of the patient's cochlea is not a current practice for CI surgery [1]. By including measured mechanical properties of the basilar membrane into our force control strategy, we can detect if the CI is about to rupture into adjacent cochlear chambers. Unexpected increases in force will be detected before they become problematic. Corrective action can be taken by adapting the guidance continuously, or by reversing the insertion for a small distance and then modifying the magnetic guidance plan.

Implementing force feedback control is complicated by the fact that the force sensor is not colocated with the implant tip. Interpreting tip forces, especially since the implant is flexible, may be difficult. That is, forces on the tip (including ST contact forces and attractive magnetic forces) may not be easily measured with a force sensor located at the base. An estimate of the maximum attractive force of $10 \mathrm{mN}$ was 
computed using (7) at the closest dipole-to-dipole distance of $29 \mathrm{~mm}$ with the maximum-field method. In the experiments of Fig. 8, the corresponding, non-axial force data $\left(F_{x}^{2}+F_{y}^{2}\right)^{1 / 2}$ never exceeded $10 \mathrm{mN}$. One study measured the force required to puncture the basilar membrane at 26$35 \mathrm{mN}$ [19]. Clearly, the attractive forces on the implant did not increase the overall friction because the insertion force $\left(\mathrm{F}_{z}\right)$ decreased with the use of magnetic guidance. Further, some attractive force may be beneficial to direct the implant towards the apex of the cochlea (Fig. 2).

Our magnetic guidance approach can be scaled according to clinical demands. The magnetic field strength of any source is homothetic, meaning that the field of a permanent magnet is unchanged as the magnet's dimensions are scaled. If we scale the magnet's linear dimensions by a factor $s$ then its volume scales as $s^{3}$. Therefore, assuming identical magnetization, the dipole strength $\mathbf{M}$ will scale as $s^{3}$. The field strength measured at a scaled distance $s|\mathbf{p}|$ away from the magnet's center is the same as the field strength measured a distance $|\mathbf{p}|$ away from the original magnet.

Progress toward clinical feasibility will require several issues to be addressed. The obvious clinical concerns regarding MRI safety can be addressed by a CI prototype design with a removable magnetic tip. Alignment of the manipulator rotation axis with the central axis of the cochlea may be difficult in practice and warrants some study on the effects of misalignment. Attractive forces will pull the implant towards the delicate basilar membrane, which, if excessive, could puncture it. However, in our implementation, the dominant magnetic torque is used to align the implant tip in the direction of the ST channel, which is parallel to the ST walls. Thus, the typical scenario of basilar perforation in which the electrode tip impinges on the basilar membrane is avoided. Our experimental setup and results used a scaled 3:1 implant prototype and ST phantom. Eventually, these experiments must be performed at 1:1 scale, requiring a $1: 1$ scale implant and a 1:1 scale ST phantom with both cochleostomy and round-window openings. Deviations into other scalar chambers could not be assessed with our experiment since trajectories were preplanned through a phantom consisting of only the ST path. Proper assessment may require a phantom with all scalar chambers separated by membranes that mimic the basilar and Reissner's membrane. In-vitro studies with ST phantoms and cadaver temporal bones directly comparing our prototype and insertion method against commercial CIs using standard techniques are required for proper evaluation.

\section{CONCLUSiON}

We have presented a proof-of-concept study that demonstrates the efficacy of implementing magnetic guidance as a clinical strategy towards improved cochlear implant surgery. By applying magnetic torque to the tip of a cochlear-implant prototype during insertions, we reduced the insertions forces by approximately $50 \%$. Such reductions will likely reduce insertion trauma by a proportional amount, and may enable deeper insertion into the cochlea for improved performance.

\section{ACKNOWLEDGMENT}

This research is part of a larger collaboration with Dr. Robert Webster at Vanderbilt University, and the authors would like to thank him for fruitful discussions.

\section{REFERENCES}

[1] S. J. Rebscher, A. Hetherington, B. Bonham, P. Wardrop, D. Whinney, and P. A. Leake, "Considerations for design of future cochlear implant electrode arrays: Electrode array stiffness, size, and depth of insertion," J. Rehabil. Res. Dev., vol. 45, no. 5, pp. 731-747, 2008.

[2] R. J. S. Briggs, M. Tykocinski, E. Saunders, W. Hellier, M. Dahm, B. Pyman, and G. M. Clark, "Surgical implications of perimodiolar cochlear implant electrode design: avoiding intracochlear damage and scala vestibuli insertion," Cochlear Implants Int., vol. 2, no. 2, pp. $135-149,2001$.

[3] O. Adunka and J. Kiefer, "Impact of electrode insertion depth on intracochlear trauma," Otolaryngol. Head Neck Surg., vol. 135, no. 3, pp. 374-382, 2006

[4] W. K. Gstoettner, O. Adunka, P. Franz, J. Hamzavi, H. Plenk, M. Susani, W. Baumgartner, and J. Kiefer, "Perimodiolar electrodes in cochlear implant surgery," Acta Otolaryngol., vol. 121, no. 2, pp. 216-219, 2001.

[5] J. T. Roland, Jr., "A model for cochlear implant electrode insertion and force evaluation: Results with a new electrode design and insertion technique," Laryngoscope, vol. 115, no. 8, pp. 1325-1339, 2005.

[6] C. A. Todd, F. Naghdy, and M. J. Svehla, "Force application during cochlear implant insertion: An analysis for improvement of surgeon technique," IEEE Trans. Biomed. Eng., vol. 54, no. 7, pp. 1247-1255, 2007.

[7] D. Schurzig, R. J. Webster, III, M. S. Dietrich, and R. F. Labadie, "Force of cochlear implant electrode insertion performed by a robotic insertion tool: comparison of traditional versus advance off-stylet techniques," Otol. Neurotol., vol. 31, no. 8, pp. 1207-1210, 2010.

[8] H. Mirzadeh and F. Abbasi, "Segmented detachable structure of Cochlear-Implant electrodes for Close-Hugging engagement with the modiolus," J. Biomed. Mater. Res. B Appl. Biomater, vol. 68B, no. 2, pp. 191-198, 2004.

[9] B. Y. Arcand, P. T. Bhatti, N. V. Butala, J. Wang, C. R. Friedrich, and K. D. Wise, "Active positioning device for a perimodiolar cochlear electrode array," Microsys.Tech., vol. 10, pp. 478-483, 2004.

[10] J. Wu, L. Yan, H. Xu, W. C. Tang, and F.-G. Zeng, "A curvaturecontrolled 3D micro-electrode array for cochlear implants," in IEEE Int. Conf. Solid St. Sens. Act. Microsys., vol. 2, 2005, pp. 1636-1639.

[11] B. Chen, H. N. Kha, and G. M. Clark, "Development of a steerable cochlear implant electrode array," in IFMBE Int. Conf. Biomed. Eng., vol. 15, 2007, pp. 607-610.

[12] J. Zhang, J. T. Roland, Jr., S. Manolidis, and N. Simaan, "Optimal path planning for robotic insertion of steerable electrode arrays in cochlear implant surgery," ASME J. Med. Devices, vol. 3, no. 011001, 2009.

[13] J. Zhang, W. Wei, J. Ding, J. T. Roland, Jr., S. Manolidis, and N. Simaan, "Inroads toward robot-assisted cochlear implant surgery using steerable electrode arrays," Otol. Neurotol., vol. 31, no. 8, pp. 1199-1206, 2010.

[14] O. Majdani, D. Schurzig, A. Hussong, T. Rau, J. Wittkopf, T. Lenarz, and R. F. Labadie, "Force measurement of insertion of cochlear implant electrode arrays in vitro: comparison of surgeon to automated insertion tool," Acta Otolaryngol., vol. 130, no. 1, pp. 31-36, 2010.

[15] D. Schurzig, R. F. Labadie, A. Hussong, T. S. Rau, and R. J. Webster, III, "Design of a tool integrating force sensing with automated insertion in cochlear implantation," IEEE/ASME Trans. Mechatronics, DOI 10.1109/TMECH.2011.2106795.

[16] J. R. Clark, F. M. Warren, and J. J. Abbott, "A scalable model for human scala-tympani phantoms," ASME J. Med. Devices, vol. 5, no. 014501, 2011.

[17] M. N. Maghribi, P. A. Krulevitch, J. C. Davidson, and J. K. Hamilton, "Implantable devices using magnetic guidance," U.S. Patent, 2006.

[18] J. J. Abbott, O. Ergeneman, M. P. Kummer, A. M. Hirt, and B. J. Nelson, "Modeling magnetic torque and force for controlled manipulation of Soft-Magnetic bodies," IEEE Trans. Robot., vol. 23, no. 6, pp. 1247-1252, 2007.

[19] T. Ishii, M. Takayama, and Y. Takahashi, "Mechanical properties of human round window, basilar and reissner's membranes." Acta Otolaryngol., vol. 115, no. s519, pp. 78-82, 1995. 